\title{
PERTURBED ASYMPTOTICALLY LINEAR PROBLEMS
}

\author{
R. BARTOLO, A.M. CANDELA, AND A. SALVATORE
}

\begin{abstract}
The aim of this paper is investigating the existence of solutions of the semilinear elliptic problem$$
\begin{cases}-\Delta u=p(x, u)+\varepsilon g(x, u) & \text { in } \Omega, \\ u=0 & \text { on } \partial \Omega,\end{cases}
$$

where $\Omega$ is an open bounded domain of $\mathbb{R}^{N}, \varepsilon \in \mathbb{R}, p$ is subcritical and asymptotically linear at infinity and $g$ is just a continuous function. Even when this problem has not a variational structure on $H_{0}^{1}(\Omega)$, suitable procedures and estimates allow us to prove that the number of distinct crtitical levels of the functional associated to the unperturbed problem is "stable" under small perturbations, in particular obtaining multiplicity results if $p$ is odd, both in the non-resonant and in the resonant case.
\end{abstract}

\section{INTRODUCTION}

Let us consider the following semilinear elliptic problem

$$
\begin{cases}-\Delta u=p(x, u)+\varepsilon g(x, u) & \text { in } \Omega, \\ u=0 & \text { on } \partial \Omega,\end{cases}
$$

where $\Omega$ is an open bounded domain of $\mathbb{R}^{N}(N \geq 3)$ with smooth boundary $\partial \Omega$, $\varepsilon \in \mathbb{R}$ and $p, g$ are given real functions on $\Omega \times \mathbb{R}$.

If $\varepsilon=0$ problem (1.1) has been widely investigated when $p(x, \cdot)$ is asymptotically linear and possibly odd (see [1,3] and references therein).

On the other hand, if $\varepsilon \neq 0$, let us consider a perturbation term $g$ just continuous, without assumptions on its growth. In [12] Li and Liu state the existence of multiple solutions of $(1.1)$ when $p(x, \cdot)$ is odd, superlinear at infinity, but subcritical (see also [9] and [13] for related results). Furthermore, in [13, Theorem 1.6] a multiplicity result is stated if $p(x, \cdot)$ is asymptotically linear at infinity and odd when $g(x, \cdot)$ is odd, too (see also Theorem 1.3 below). Here, our aim is going further in this direction and proving that, even when (1.1) has not a variational structure on $H_{0}^{1}(\Omega)$, the number of distinct critical levels of the functional associated to the unperturbed problem is "stable" under small perturbations also in lack of symmetry.

Throughout this paper suppose that there exist $\lambda \in \mathbb{R}$ and $f: \Omega \times \mathbb{R} \rightarrow \mathbb{R}$ such that

$$
p(x, t)=\lambda t+f(x, t) \quad \text { for all } t \in \mathbb{R} \text { and a.e. } x \in \Omega .
$$

Denote respectively by $\sigma(-\Delta)$ and by $0<\lambda_{1}<\lambda_{2}<\ldots<\lambda_{k}<\ldots$ the spectrum and the distinct eigenvalues of $-\Delta$ in $H_{0}^{1}(\Omega)$.

2000 Mathematics Subject Classification. 35J20, 58E05.

Key words and phrases. Asymptotically linear elliptic problem, essential value, perturbed problem, variational methods, pseudo-genus, resonant problem.

Partially supported by M.I.U.R. Research project PRIN2009 "Metodi Variazionali e Topologici nello Studio di Fenomeni Nonlineari". 
Let us introduce the following conditions:

$\left(H_{0}\right) \quad f$ is a Carathéodory function (i.e. $f(\cdot, t)$ is measurable in $\Omega$ for all $t \in \mathbb{R}$ and $f(x, \cdot)$ is continuous in $\mathbb{R}$ for a.e. $x \in \Omega)$ and

$$
\sup _{|t| \leq r}|f(\cdot, t)| \in L^{\infty}(\Omega) \quad \text { for all } \quad r>0
$$

$\left(H_{1}\right)$ there exists

$$
\lim _{|t| \rightarrow+\infty} \frac{f(x, t)}{t}=0 \quad \text { uniformly with respect to a.e. } x \in \Omega
$$

$\left(H_{2}\right)$ there exists

$$
\lim _{t \rightarrow 0} \frac{f(x, t)}{t}=: f^{\prime}(0) \in \mathbb{R} \quad \text { uniformly with respect to a.e. } x \in \Omega ;
$$

$\left(H_{3}\right) \lambda \notin \sigma(-\Delta)$;

$\left(H_{4}\right)$ there exist two integers $h, k \geq 1$ such that

$$
\min \left\{f^{\prime}(0)+\lambda, \lambda\right\}<\lambda_{h}<\lambda_{k}<\max \left\{f^{\prime}(0)+\lambda, \lambda\right\}
$$

$\left(H_{5}\right) f(x, \cdot)$ is odd for a.e. $x \in \Omega$.

By (1.2) problem (1.1) becomes

$$
\begin{cases}-\Delta u-\lambda u=f(x, u)+\varepsilon g(x, u) & \text { in } \Omega, \\ u=0 & \text { on } \partial \Omega .\end{cases}
$$

If $\left(H_{0}\right)-\left(H_{1}\right)$ hold and $\varepsilon=0$, it is well known that the solutions of problem $\left(P_{0}\right)$ are the critical points of the $C^{1}$ functional

$$
I(u)=\frac{1}{2} \int_{\Omega}|\nabla u|^{2} \mathrm{~d} x-\frac{\lambda}{2} \int_{\Omega} u^{2} \mathrm{~d} x-\int_{\Omega} F(x, u) \mathrm{d} x \quad \text { on } \quad H_{0}^{1}(\Omega),
$$

with $F(x, t)=\int_{0}^{t} f(x, s) \mathrm{d} s$. By using the pseudo-index theory introduced by Benci in [4], it can be proven that $I$ has at least as many distinct critical points as the number of the eigenvalues of $-\Delta$ between $f^{\prime}(0)+\lambda$ and $\lambda$, even if some of the corresponding critical levels may be the same (cf. [3] and, here, Section 3).

In our first theorem we give an existence result for $\left(P_{\varepsilon}\right)$ and, in the easier case in which both $f$ and the pertubation function $g$ are odd, we obtain also multiplicity of solutions: the crucial point is that, in this case, near critical values of $I$ there are critical values of odd perturbations of $I$ (see Theorem 2.5).

Theorem 1.1. Assume that $g \in C(\bar{\Omega} \times \mathbb{R}, \mathbb{R})$ and $\left(H_{0}\right),\left(H_{1}\right)$ and $\left(H_{3}\right)$ hold. Then, there exists $\bar{\varepsilon}>0$ such that problem $\left(P_{\varepsilon}\right)$ has at least one solution for all $|\varepsilon| \leq \bar{\varepsilon}$. Moreover, if $g$ is odd, under the further assumptions $\left(H_{2}\right),\left(H_{4}\right)$ and $\left(H_{5}\right)$, denoting by $\bar{m}$, with $1 \leq \bar{m} \leq \operatorname{dim}\left(M_{h} \oplus \ldots \oplus M_{k}\right)$, the number of the distinct mini-max critical levels of the unperturbed functional $I$ in $(1.4)$, then problem $\left(P_{\varepsilon}\right)$ has at least $\bar{m}$ distinct pairs of solutions for all $|\varepsilon| \leq \bar{\varepsilon}$.

We point out that assumption $\left(H_{3}\right)$ can be avoided. Indeed, many authors studied problem $\left(P_{0}\right)$ in the so called "resonant case", but under suitable stronger hypotheses on the nonlinear term $f$ (see [3] and references therein). Here, we consider the following further conditions:

$\left(H_{1}^{\prime}\right)$ there exists $M>0$ such that

$$
|f(x, t)| \leq M \quad \text { for a.e. } x \in \Omega \text { and for all } t \in \mathbb{R}
$$


$\left(H_{3}^{\prime}\right)$ there exists an integer $k \geq 1$ such that $\lambda=\lambda_{k} \in \sigma(-\Delta)$;

$\left(H_{4}^{\prime}\right)$ there exists an integer $h \geq 1$ such that

$$
\min \left\{f^{\prime}(0)+\lambda_{k}, \lambda_{k}\right\}<\lambda_{h}<\max \left\{f^{\prime}(0)+\lambda_{k}, \lambda_{k}\right\}
$$

$\left(H_{6}\right)$ there exists

$$
\lim _{|t| \rightarrow+\infty} F(x, t)=l \in\{ \pm \infty\} \quad \text { uniformly with respect to a.e. } x \in \Omega .
$$

Theorem 1.2. Assume that $g \in C(\bar{\Omega} \times \mathbb{R}, \mathbb{R})$ and $\left(H_{0}\right),\left(H_{1}^{\prime}\right),\left(H_{3}^{\prime}\right)$ and $\left(H_{6}\right)$ hold. Then there exists $\bar{\varepsilon}>0$ such that problem $\left(P_{\varepsilon}\right)$ has at least one solution for all $|\varepsilon| \leq \bar{\varepsilon}$.

Moreover, if $g$ is odd, under the further assumptions $\left(H_{2}\right),\left(H_{4}^{\prime}\right)$ and $\left(H_{5}\right)$, the same multiplicity result of Theorem 1.1 holds.

We point out that in [13, Theorem 1.6] the authors obtain a result sharper than our in Theorem 1.2, but it works only in the non resonant case. Furthermore, our approach allows us to deal also with the case in which the symmetry of problem $\left(P_{\varepsilon}\right)$ is broken by a non-odd perturbation term $g$. In this case, we still obtain some multiplicity results, but our techniques impose to work with nonlinearities such that the functional $I$ in (1.4) has only critical levels which arise for topological reasons, called topologically relevant (see Definition 2.10). Anyway, similar assumptions appear also in previous references concerning asymptotically linear (unperturbed) Dirichlet problems, as different approaches require sometimes that the critical points of the associated functional are "nondegenerate" (see e.g. [6] and references therein). Roughly speaking, we need to restrict a priori the set of the critical values of our functional $I$ in (1.4), so that they are indeed essential ones (cf. Definition 2.4) and a stability result, stating that critical values of $I$ are preserved for small perturbations, holds (see Corollary 2.11, while we refer to [14, Theorem 8.10] for a related result based on Morse Theory). In [16] Reeken obtains something stronger. Indeed, he gives a topological description of some critical levels $c$ of a $C^{1}$ functional and proves that the category of the set of the critical points of small perturbations of the functional is lower bounded from an integer representing the topological description of $c$ (see [16, Theorem 5.2]).

Theorem 1.3. Assume that $g \in C(\bar{\Omega} \times \mathbb{R}, \mathbb{R})$ and $\left(H_{0}\right)-\left(H_{5}\right)$ hold. If all the critical levels of the functional I in (1.4) are topologically relevant, denoting by $\bar{m}$, with $1 \leq \bar{m} \leq \operatorname{dim}\left(M_{h} \oplus \ldots \oplus M_{k}\right)$, the number of the distinct mini-max critical levels of $I$, then problem $\left(P_{\varepsilon}\right)$ has at least $\bar{m}$ distinct solutions for all $|\varepsilon| \leq \bar{\varepsilon}$.

We can extend the previous theorem to the resonant case as follows.

Theorem 1.4. Under the assumptions of Theorem 1.3, just replacing hypotheses $\left(H_{1}\right),\left(H_{3}\right),\left(H_{4}\right)$ respectively by $\left(H_{1}^{\prime}\right),\left(H_{3}^{\prime}\right),\left(H_{4}^{\prime}\right)$ and adding condition $\left(H_{6}\right)$, the same multiplicity result of Theorem 1.3 holds also in the resonant case.

Remark 1.5. The previous results hold also if in assumption $\left(H_{2}\right)$ it is $f^{\prime}(0)=0$. Indeed, in this case $\left(H_{4}\right)$ is not meaningful, but imposing a condition on the sign of $F$, a multiplicity result can still be stated (see Remarks 3.2 and 4.1). On the other hand, our theorems can be also proven when the limit in $\left(H_{2}\right)$ is infinite, i.e. $f^{\prime}(0) \in\{ \pm \infty\}$ (see Remarks 3.3 and 4.1 for more details).

This paper is organized as follows. In Section 2 we recall some abstract tools, in particular concerning the notions of pseudo-index and essential value, in Section 3 
we deal with the unperturbed problem $\left(P_{0}\right)$ and, lastly, in Section 4 we prove our main results.

Notations. Throughout this paper we denote by $\left(X,\|\cdot\|_{X}\right)$ a Banach space, by $\left(X^{\prime},\|\cdot\|_{X^{\prime}}\right)$ its dual, by $J$ a $C^{1}$ functional on $X$ and by

- $J^{b}=\{u \in X: J(u) \leq b\}$ the sublevel of $J$ corresponding to $b \in \overline{\mathbb{R}}:=$ $\mathbb{R} \cup\{ \pm \infty\}$

- $K_{c}=\{u \in X: J(u)=c, \mathrm{~d} J(u)=0\}$ the set of the critical points of $J$ in $X$ at the critical level $c \in \mathbb{R}$.

Furthermore, let us denote by

- $|\cdot|_{s}$ the usual norm in the Lebesgue space $L^{s}(\Omega), 1 \leq s \leq+\infty$;

- $\|\cdot\|$ the norm in $H_{0}^{1}(\Omega)$, i.e. $\|u\|=|\nabla u|_{2}$ for all $u \in H_{0}^{1}(\Omega)$;

- $2^{*}=\frac{2 N}{N-2}$ the critical exponent for Sobolev embeddings of $H_{0}^{1}(\Omega)(N \geq 3)$;

- $M_{j}$ the eigenspace corresponding to the eigenvalue $\lambda_{j}$ of $-\Delta$ in $H_{0}^{1}(\Omega)$ and $u_{j}$ the component of $u$ in $M_{j}$, for any integer $j \geq 1$ and for each $u \in H_{0}^{1}(\Omega)$;

- $H^{-}(j)=\bigoplus_{i \leq j} M_{i}$ and $H^{+}(j)=\overline{\bigoplus_{i \geq j} M_{i}}$, for any integer $j \geq 1$;

- $C_{j}$ a positive real number, for any integer $j \geq 1$.

\section{Some abstract tools}

We recall the well-known Palais-Smale condition.

Definition 2.1. The functional $J$ satisfies the Palais-Smale Condition at level $c$ $(c \in \mathbb{R})$, briefly $(P S)_{c}$, if any sequence $\left(u_{n}\right)_{n} \subset X$ such that

$$
\lim _{n \rightarrow+\infty} J\left(u_{n}\right)=c \quad \text { and } \quad \lim _{n \rightarrow+\infty}\left\|\mathrm{d} J\left(u_{n}\right)\right\|_{X^{\prime}}=0,
$$

converges in $X$, up to subsequences. In general, if $-\infty \leq a<b \leq+\infty, J$ satisfies $(P S)$ in $] a, b[$ if so is at each level $c \in] a, b[$.

Beside the existence critical point theorems, under this condition sharper results can be proven when one deals with symmetric functionals (see e.g. [2]). In [3] multiplicity results for critical points of even functionals are stated and their proofs are based on the use of a pseudo-index theory. In order to introduce such definition, let us recall some notions of the index theory for an even functional with symmetry $\operatorname{group} \mathbb{Z}_{2}=\{\mathrm{id},-\mathrm{id}\}$.

Define

$$
\begin{gathered}
\Sigma=\Sigma(X)=\{A \subset X: A \text { closed and symmetric w.r.t. the origin, } \\
\text { i.e. }-u \in A \text { if } u \in A\}
\end{gathered}
$$

and

$$
\mathcal{H}=\{h \in C(X, X): h \text { odd }\} .
$$

Taking $A \in \Sigma, A \neq \emptyset$, the genus of $A$ is

$$
\gamma(A)=\inf \left\{k \in \mathbb{N}^{*}: \exists \psi \in C\left(A, \mathbb{R}^{k} \backslash\{0\}\right) \text { s.t. } \psi(-u)=-\psi(u) \text { for all } u \in A\right\},
$$

if such an infimum exists, otherwise $\gamma(A)=+\infty$. Assume $\gamma(\emptyset)=0$.

The index theory $(\Sigma, \mathcal{H}, \gamma)$ related to $\mathbb{Z}_{2}$ is also called genus (see [10] and for more details [17, Section II.5]). 
According to [4], the pseudo-index related to the genus, an even functional $J: X \rightarrow \mathbb{R}$ and $S \in \Sigma$ is the triplet $\left(S, \mathcal{H}^{*}, \gamma^{*}\right)$ such that

$$
\mathcal{H}^{*}=\left\{h \in \mathcal{H}: h \text { bounded homeomorphism s.t. } h(u)=u \text { if } u \notin J^{-1}(] 0,+\infty[)\right\}
$$

and

$$
\gamma^{*}(A)=\min _{h \in \mathcal{H}^{*}} \gamma(h(A) \cap S) \text { for all } A \in \Sigma .
$$

A mini-max theorem was stated in [3, Theorem 2.9] under the weaker Cerami's variant of the Palais-Smale condition (cf. e.g. [3, Definition 1.1]), while here we recall it when just the $(P S)$ condition holds.

Theorem 2.2. Let $H$ be a real Hilbert space, $J \in C^{1}(H, \mathbb{R})$ an even functional, $(\Sigma, \mathcal{H}, \gamma)$ the genus theory on $H, S \in \Sigma,\left(S, \mathcal{H}^{*}, \gamma^{*}\right)$ the pseudo-index theory related to the genus, $J$ and $S$. Consider $a, b, c_{0}, c_{\infty} \in \overline{\mathbb{R}},-\infty \leq a<c_{0}<c_{\infty}<b \leq+\infty$. Assume that:

(i) the functional $J$ satisfies $(P S)$ in $] a, b[$;

(ii) $S \subset J^{-1}\left(\left[c_{0},+\infty[)\right.\right.$;

(iii) there exist an integer $\bar{k} \geq 1$ and $\bar{A} \in \Sigma$ such that $\bar{A} \subset J^{c_{\infty}}$ and $\gamma^{*}(\bar{A}) \geq \bar{k}$.

Then the numbers

$$
c_{i}=\inf _{A \in \Sigma_{i}} \sup _{u \in A} J(u), \quad i \in\{1, \ldots, \bar{k}\},
$$

with $\Sigma_{i}=\left\{A \in \Sigma: \gamma^{*}(A) \geq i\right\}$, are critical values for $J$ and

$$
c_{0} \leq c_{1} \leq \ldots \leq c_{\bar{k}} \leq c_{\infty} .
$$

Furthermore, if $c=c_{i}=\ldots=c_{i+r}$, with $i \geq 1$ and $i+r \leq \bar{k}$, then $\gamma\left(K_{c}\right) \geq r+1$.

We have already pointed out that in our main theorems we may deal with problems $\left(P_{\varepsilon}\right)$ without a variational structure on $H_{0}^{1}(\Omega)$. Hence, following [12], we use the auxiliary notion of essential value, as it is introduced in [8] (see also [7]) in the study of perturbations of nonsmooth functionals. Moreover, for even functionals we introduce the definition of odd-essential value, which allows us to obtain multiplicity results for odd perturbations.

Definition 2.3. Let $J: X \rightarrow \mathbb{R}$ be continuous (resp. $J$ even continuous) and $a, b \in \overline{\mathbb{R}}$, with $a \leq b$. The pair $\left(J^{b}, J^{a}\right)$ is trivial (resp. odd-trivial) if, for each neighbourhood $\left[\alpha^{\prime}, \alpha^{\prime \prime}\right]$ of $a$ and $\left[\beta^{\prime}, \beta^{\prime \prime}\right]$ of $b$ in $\overline{\mathbb{R}}$, there exists a continuous (resp. an odd continuous) map $\varphi: J^{\beta^{\prime}} \times[0,1] \rightarrow J^{\beta^{\prime \prime}}$ such that

(i) $\varphi(x, 0)=x$ for each $x \in J^{\beta^{\prime}}$;

(ii) $\varphi\left(J^{\beta^{\prime}} \times\{1\}\right) \subseteq J^{\alpha^{\prime \prime}}$

(iii) $\varphi\left(J^{\alpha^{\prime}} \times[0,1]\right) \subseteq J^{\alpha^{\prime \prime}}$.

Definition 2.4. Let $J: X \rightarrow \mathbb{R}$ be continuous (resp. $J$ even continuous). A real number $c$ is an essential value (resp. an odd-essential value) of $J$ if for each $\varepsilon>0$ there exist $a, b \in] c-\varepsilon, c+\varepsilon\left[, a<b\right.$, such that the pair $\left(J^{b}, J^{a}\right)$ is not trivial (resp. not odd-trivial).

The following theorem states that small perturbations of a continuous functional preserve the essential values (see [8, Theorem 3.1] or also [7, Theorem 2.6]); in particular this holds for the odd ones, just doing some small changes in the proof of [8, Theorem 3.1]. 
Theorem 2.5. Let $c \in \mathbb{R}$ be an essential value (resp. odd-essential value) of $J: X \rightarrow \mathbb{R}$ continuous (resp. J even continuous). Then, for every $\eta>0$ there exists $\delta>0$ such that every functional (resp. even functional) $G \in C(X, \mathbb{R})$ with

$$
\sup \{|J(u)-G(u)|: u \in X\}<\delta
$$

admits an essential value (resp. odd-essential value) in $] c-\eta, c+\eta[$.

Now, we focus on the setting of smooth functionals and recall some results which link critical and essential values, stating in particular that the critical values arising from mini-max procedures are essential, if all the involved deformations are of the "same kind" (see [8, Theorems 3.7 and 3.9]).

Theorem 2.6. Let $c \in \mathbb{R}$ be an essential value of $J \in C^{1}(X, \mathbb{R})$. If $(P S)_{c}$ holds, then $c$ is a critical value of $J$.

Remark 2.7. In general the reverse implication does not hold: even if $(P S)_{c}$ is satisfied, a critical value is not necessarily an essential one (see e.g. [8, Example $3.12])$.

Theorem 2.8. Let $\Gamma$ be a non empty family of non empty subsets of $X, J \in$ $C^{1}(X, \mathbb{R})$ and $d \in \mathbb{R} \cup\{-\infty\}$. Let us assume that, for every $C \in \Gamma$ and for every deformation $\varphi: X \times[0,1] \longrightarrow X$ with $\varphi(u, t)=u$ on $J^{d} \times[0,1]$, it is $\overline{\varphi(C \times\{1\})} \in \Gamma$. Setting

$$
c=\inf _{C \in \Gamma} \sup _{u \in C} J(u)
$$

if $d<c<+\infty$, then $c$ is an essential value of $J$.

Let us point out that, if $J$ is even, the previous theorem does not apply to the critical values $c_{i}$ given by (2.1); in fact, if $\varphi$ is a deformation as in Theorem 2.8, the set $\overline{\varphi(C \times\{1\})}, C \in \Gamma$, does not necessarily belong to $\Gamma$ because $\varphi$ could be not odd. Thus, we cannot assert that the $c_{i}$ 's are indeed essential values of $J$. Anyway, slight modifications in the proof of [8, Theorem 3.9] allow us to state the following result concerning odd-essential values.

Corollary 2.9. Let $\Gamma$ be a non empty family of non empty symmetric subsets of $X, J \in C^{1}(X, \mathbb{R})$ even and $d \in \mathbb{R} \cup\{-\infty\}$. Let us assume that, for every $C \in \Gamma$ and for every odd deformation $\varphi: X \times[0,1] \longrightarrow X$ with $\varphi(u, t)=u$ on $J^{d} \times[0,1]$, it is $\overline{\varphi(C \times\{1\})} \in \Gamma$.

Then, taking $c$ as in (2.2), if $d<c<+\infty$, we have that $c$ is an odd-essential value of $J$.

As we have observed in the Introduction, we also deal with perturbation from symmetry problems, thus we restrict ourselves to consider a subset of the critical values of $I$ in (1.4), so that a stability result does still hold (we also refer to [11] for a related result concerning changing-sign solutions of some elliptic equations).

Actually, in Corollary 2.11 below we consider just the preservation for small perturbations of some critical levels of a smooth functional satisfying the $(P S)$ condition. ¿From the Deformation Lemma, if $J \in C^{1}(X, \mathbb{R})$ satisfies $(P S)_{c}$ and $c$ is not a critical value, then for any $\bar{\eta}>0$ there exists $\eta \in] 0, \bar{\eta}[$ and $\varphi \in C(X \times[0,1], X)$, which is odd if $J$ is even, such that $\varphi(u, 1)=u$ if $J(u) \notin[c-\bar{\eta}, c+\bar{\eta}]$ and $\varphi\left(J^{c+\eta}, 1\right) \subset$ $J^{c-\eta}$. In some sense now we require that also the other implication is true, that is if $J^{c-\eta}$ is a strong deformation retract of $J^{c+\eta}$, then $c$ is not critical.

Indeed, starting from Definitions 2.3 and 2.4, we give the following definition. 
Definition 2.10. A critical level $c$ of a functional $J \in C^{1}(X, \mathbb{R})$ is topologically relevant if it is an essential one.

Summing up, we work with a special class of critical levels: those which are also essential.

Thus, according to Definition 2.10, we point out the following consequence of Theorems 2.5 and 2.6.

Corollary 2.11. Let $c \in \mathbb{R}$ be a topologically relevant critical value of a functional $J \in C^{1}(X, \mathbb{R})$. Then, for every $\eta>0$ there exists $\delta>0$ such that every functional $G \in C^{1}(X, \mathbb{R})$ satisfying $(P S)$ in $] c-\eta, c+\eta[$ with

$$
\sup \{|J(u)-G(u)|: u \in X\}<\delta
$$

admits a critical value in $] c-\eta, c+\eta[$.

\section{The SYMmetric CASE}

In this section we deal with some existence and multiplicity results about the unperturbed problem

$$
\begin{cases}-\Delta u-\lambda u=f(x, u) & \text { in } \Omega, \\ u=0 & \text { on } \partial \Omega .\end{cases}
$$

In previous references on this topic, problem $\left(P_{0}\right)$ has been mainly studied when $f=f(u)$ or $f=f(x, u) \in C(\bar{\Omega} \times \mathbb{R}, \mathbb{R})$; instead here we deal with a Carathéodory nonlinearity $f$ only measurable on $x$, thus we need a global control on its growth.

Remark that from $\left(H_{0}\right),\left(H_{1}\right)$ and direct computations it follows that for all $\sigma>0$ there exists $A_{\sigma}>0$ such that

$$
|f(x, t)| \leq \sigma|t|+A_{\sigma} \quad \text { for all } t \in \mathbb{R} \text { and a.e. } x \in \Omega .
$$

Thus, the functional $I$ defined in (1.4) is $C^{1}$ (cf. e.g. [17, Appendix C]). Hence, the weak solutions of problem $\left(P_{0}\right)$ are the critical points of $I$. Let us point out that this is true even if, instead of (1.3), we only require that

$$
\sup _{|t| \leq r}|f(\cdot, t)| \in L^{\frac{2 N}{N+2}}(\Omega) \quad \text { for all } \quad r>0
$$

since $\frac{2 N}{N+2}$ is the conjugate exponent of $2^{*}$.

In the unperturbed non resonant case, the following result holds.

Theorem 3.1. Assume that $\left(H_{0}\right),\left(H_{1}\right)$ and $\left(H_{3}\right)$ hold. Then, problem $\left(P_{0}\right)$ has at least one solution. Moreover, under the further assumptions $\left(H_{2}\right),\left(H_{4}\right)$ and $\left(H_{5}\right)$, problem $\left(P_{0}\right)$ has at least $\operatorname{dim}\left(M_{h} \oplus \ldots \oplus M_{k}\right)$ distinct pairs of nontrivial solutions.

Proof. Let us point out that by $\left(H_{3}\right)$ functional $I$ satisfies $(P S)$ in $\mathbb{R}$ (cf. e.g. [1]). Hence, a standard application of the saddle point theorem (see [15, Theorem 4.6]) allows us to prove the existence of one solution for $\left(P_{0}\right)$.

Here, we focus on the multiplicity statement concerning the critical points of $I$, which is even by assumption $\left(H_{5}\right)$.

At first we restrict to the case $f^{\prime}(0)<0$. Hence, without loss of generality, in $\left(H_{4}\right)$ we can assume that $h$ and $k$ are such that

$$
\lambda_{h-1}<f^{\prime}(0)+\lambda<\lambda_{h}<\lambda_{k}<\lambda<\lambda_{k+1},
$$


possibly with $\lambda_{0}=-\infty$. Notice that by (3.1), fixing any $\sigma>0, B_{\sigma}>0$ exists such that

$$
I(u) \leq \frac{1}{2}\|u\|^{2}-\frac{\lambda}{2}|u|_{2}^{2}+\frac{\sigma}{2}|u|_{2}^{2}+B_{\sigma}|u|_{2} \quad \text { for all } u \in H_{0}^{1}(\Omega) .
$$

Then, it results

$$
I(u) \leq \frac{1}{2} \sum_{j=1}^{k}\left(\lambda_{j}-\lambda+\sigma\right)\left|u_{j}\right|_{2}^{2}+B_{\sigma}|u|_{2} \quad \text { for all } u=\sum_{j=1}^{k} u_{j} \in H^{-}(k) .
$$

Hence, taking $\sigma$ small enough, by (3.2) the functional $I$ tends to $-\infty$ as $\|u\|$ diverges in $H^{-}(k)$, so there exists $c_{\infty} \in \mathbb{R}$ such that $I(u) \leq c_{\infty}$ for all $u \in H^{-}(k)$.

Next we need a control on $F$ near to $t=0$. ¿From $\left(H_{1}\right)$ and $\left(H_{2}\right)$, for any $\sigma>0$ there exist $R_{\sigma}, \delta_{\sigma}>0$ (without loss of generality $R_{\sigma} \geq 1$ ) such that

$$
\begin{aligned}
& |F(x, t)| \leq \frac{\sigma}{2}|t|^{2} \quad \text { if }|t|>R_{\sigma}, \text { for a.e. } x \in \Omega, \\
& \left|F(x, t)-\frac{f^{\prime}(0)}{2} t^{2}\right| \leq \frac{\sigma}{2}|t|^{2} \quad \text { if }|t|<\delta_{\sigma}, \text { for a.e. } x \in \Omega .
\end{aligned}
$$

Moreover, taking any $s \in\left[0,2^{*}-2\left[\right.\right.$, by (1.3) there exists $a_{R_{\sigma}}>0$ such that, if $\delta_{\sigma} \leq|t| \leq R_{\sigma}$ and for a.e. $x \in \Omega$, we have

$$
|F(x, t)| \leq \sup _{|t| \leq R_{\sigma}}|f(x, t)| R_{\sigma} \leq a_{R_{\sigma}} R_{\sigma} \leq a_{R_{\sigma}} R_{\sigma}\left(\frac{|t|}{\delta_{\sigma}}\right)^{s+2} .
$$

Summing up, for any $\sigma>0$ there exists $a_{\sigma}>0$ large enough such that for all $t \in \mathbb{R}$ and for a.e. $x \in \Omega$ we have

$$
-\frac{\left(\sigma-f^{\prime}(0)\right)}{2}|t|^{2}-a_{\sigma}|t|^{s+2} \leq F(x, t) \leq \frac{\left(\sigma+f^{\prime}(0)\right)}{2}|t|^{2}+a_{\sigma}|t|^{s+2},
$$

which in particular implies

$$
\int_{\Omega} F(x, u) \mathrm{d} x \leq \frac{\left(\sigma+f^{\prime}(0)\right)}{2}|u|_{2}^{2}+a_{\sigma}|u|_{s+2}^{s+2} \quad \text { for all } u \in H_{0}^{1}(\Omega) .
$$

By the Sobolev inequalities it results

$$
I(u) \geq \frac{1}{2}\left(\|u\|^{2}-\left(\lambda+f^{\prime}(0)+\sigma\right)|u|_{2}^{2}\right)-a_{\sigma}^{\prime}\|u\|^{s+2} \quad \text { for all } u \in H_{0}^{1}(\Omega),
$$

for a suitable $a_{\sigma}^{\prime}>0$. On the other hand, if $u=\sum_{j=h}^{+\infty} u_{j} \in H^{+}(h),(3.4)$ implies

$$
I(u) \geq \frac{1}{2} \sum_{j=h}^{+\infty}\left(\lambda_{j}-\left(\lambda+f^{\prime}(0)+\sigma\right)\right)\left|u_{j}\right|_{2}^{2}-a_{\sigma}^{\prime}\|u\|^{s+2},
$$

hence, by (3.2) and for $\sigma$ small enough, there exists $a_{\sigma}^{\prime \prime}>0$ such that

$$
I(u) \geq a_{\sigma}^{\prime \prime}\|u\|^{2}-a_{\sigma}^{\prime}\|u\|^{s+2} \text { for all } u \in H^{+}(h) .
$$

So, setting $S_{\rho}=\left\{u \in H_{0}^{1}(\Omega):\|u\|=\rho\right\}$, if $\rho$ is small enough there exists $c_{0}>0$ such that $I(u) \geq c_{0}$ for all $u \in S_{\rho} \cap H^{+}(h)$.

Moreover, considering the pseudo-index theory $\left(S_{\rho} \cap H^{+}(h), \mathcal{H}^{*}, \gamma^{*}\right)$ related to the genus, $S_{\rho} \cap H^{+}(h)$ and $I$, we have

$$
\gamma^{*}\left(H^{-}(k)\right)=\min _{h \in \mathcal{H}^{*}} \gamma\left(H^{-}(k) \cap h^{-1}\left(S_{\rho} \cap H^{+}(h)\right)\right) \geq \operatorname{dim} H^{-}(k)-\operatorname{codim} H^{+}(h)
$$


(cf. [3, Theorem A.2]). Hence, Theorem 2.2 applies with $\bar{A}:=H^{-}(k)$ and $S:=$ $S_{\rho} \cap H^{+}(h)$, so $I$ has at least $\operatorname{dim}\left(M_{h} \oplus \ldots \oplus M_{k}\right)$ distinct pairs of critical points corresponding to at most $\operatorname{dim}\left(M_{h} \oplus \ldots \oplus M_{k}\right)$ distinct critical values $c_{i}$, where $c_{i}$ is as in (2.1).

If $f^{\prime}(0)>0$, using (3.3), the proof follows by applying Theorem 2.2 to the functional $-I$ with $\bar{A}=: H^{+}(h)$ and $S:=S_{\rho} \cap H^{-}(k)$. Let us point out that in this case $H^{+}(h)$ is infinite dimensional, then by [5, Theorem 3.4] it is

$$
\begin{aligned}
\gamma^{*}\left(H^{+}(h)\right) & \geq \operatorname{dim}\left(H^{-}(k) \cap H^{+}(h)\right)-\operatorname{codim}\left(H^{-}(k)+H^{+}(h)\right) \\
& =\operatorname{dim}\left(M_{h} \oplus \ldots \oplus M_{k}\right),
\end{aligned}
$$

thus the proof is complete.

Remark 3.2. If in assumption $\left(H_{2}\right)$ it is $f^{\prime}(0)=0$, replacing assumption $\left(H_{4}\right)$ by

$$
F(x, t)<0 \quad \text { for a.e. } \quad x \in \Omega \text { and } t \neq 0,
$$

and reasoning as in [3, Theorem 6.1], it results $I(u) \geq c_{0}$ for all $u \in S_{\rho} \cap H^{+}(k)$. Hence we obtain $\operatorname{dim} H^{-}(k)-\operatorname{codim} H^{+}(k)=\operatorname{dim} M_{k}$ distinct pairs of critical points.

Remark 3.3. The multiplicity result stated in Theorem 3.1 still holds if the limit in assumption $\left(H_{2}\right)$ is infinite. More precisely, if the assumptions $\left(H_{0}\right),\left(H_{1}\right),\left(H_{3}\right)$, $\left(H_{5}\right)$ hold and moreover we assume

$\left(H_{7}\right)$ there exists

$$
\lim _{t \rightarrow 0} \frac{f(x, t)}{t}=-\infty \quad \text { uniformly for a.e. } x \in \Omega \text { and } \lambda_{k}<\lambda \text { for some } k \in \mathbb{N},
$$

then $\left(P_{0}\right)$ has at at least $\operatorname{dim}\left(M_{1} \oplus \ldots \oplus M_{k}\right)$ distinct pairs of non-trivial solutions, choosing in Theorem $2.2 \bar{A}=H^{-}(k)$ and $S=S_{\rho} \cap H^{+}(1)$, i.e. $S=S_{\rho}$.

On the other hand, if $\left(H_{7}\right)$ above is replaced by

$\left(H_{7}^{\prime}\right)$ there exists

$$
\lim _{t \rightarrow 0} \frac{f(x, t)}{t}=+\infty \quad \text { uniformly for a.e. } x \in \Omega,
$$

then $\left(P_{0}\right)$ has infinitely many pairs of non-trivial solutions. Indeed, fixing $h$ such that $\lambda_{h}>\lambda$, for any $k>h$ we can apply Theorem 2.2 to the functional $-I$ with $\bar{A}=H^{+}(h)$ and $S=S_{\rho} \cap H^{-}(k)$, thus obtaining $\operatorname{dim}\left(M_{h} \oplus \ldots \oplus M_{k}\right)$ pairs of solutions. The conclusion follows by the arbitrariness of $k$.

Remark 3.4. Theorem 3.1 holds also in the resonant case, with assumption $\left(H_{1}\right)$ replaced by the stronger one $\left(H_{1}^{\prime}\right)$ and $\left(H_{3}\right),\left(H_{4}\right)$ replaced respectively by $\left(H_{3}^{\prime}\right)$, $\left(H_{4}^{\prime}\right)$, but adding $\left(H_{6}\right)$ (see also [15, Theorem 4.12]). Moreover, the arguments in Remarks 3.2 and 3.3 still work.

\section{Proof of the Main Results}

Proof of Theorem 1.1. Here, we prove only the multiplicity result, since simpler arguments give the existence of one solution. Indeed, by the first statement in Theorem 3.1 we find a critical point of the unperturbed functional $I$ in (1.4) and by Theorem 2.8 the corresponding critical value is an essential one of $I$, hence by Theorems 2.5 and 2.6 the thesis follows. 
Assume that $f^{\prime}(0)<0$ and $(3.2)$ holds (similar arguments work if $f^{\prime}(0)>0$ ). Fixing any $j \in \mathbb{N}$, as in [12] we consider a continuous cut function

$$
\beta_{j}(t)=\left\{\begin{array}{lll}
0 & \text { if } & |t| \geq j+1 \\
1 & \text { if } \quad|t| \leq j
\end{array}\right.
$$

such that $0<\beta_{j}(t)<1$ if $j<|t|<j+1$. Then, let us set

$$
g_{j}(x, t)=\beta_{j}(t) g(x, t) \quad \text { and } \quad G_{j}(x, t)=\int_{0}^{t} g_{j}(x, s) \mathrm{d} s .
$$

Let us remark that, if $g(x, \cdot)$ is odd, then choosing $\beta_{j}$ even, it results that $g_{j}(x, \cdot)$ and $G_{j}(x, \cdot)$ are odd and even respectively, for a.e. $x \in \Omega$. Furthermore, there exists $\varepsilon_{1}(j)>0$ such that

$$
\varepsilon_{1}(j)\left|g_{j}(x, t)\right|<1, \quad \varepsilon_{1}(j)\left|G_{j}(x, t)\right|<1 \quad \text { for all } x \in \Omega, t \in \mathbb{R},
$$

thus for any $|\varepsilon| \leq \varepsilon_{1}(j)$ we consider the functionals

$$
I_{j, \varepsilon}(u)=I(u)-\varepsilon \int_{\Omega} G_{j}(x, u) \mathrm{d} x \quad \text { on } H_{0}^{1}(\Omega) .
$$

Let $\bar{m}$ be the number of the distinct critical levels $c_{i}$ of $I$ found in Theorem 3.1. Clearly $1 \leq \bar{m} \leq \operatorname{dim}\left(M_{h} \oplus \ldots \oplus M_{k}\right)$ and $0<c_{0}<c_{i_{1}}<\ldots<c_{i_{\bar{m}}} \leq c_{\infty}$, where $c_{0}$ and $c_{\infty}$ are as in the proof of Theorem 3.1. These critical levels are also odd-essential ones for $I$. Indeed, in order to prove this it suffices to apply Corollary 2.9. Namely, we take $X=H_{0}^{1}(\Omega), \Gamma=\Sigma_{i}$ defined in Theorem 2.2, $d=0$. Then, for any odd homeomorphism $\varphi: H_{0}^{1}(\Omega) \times[0,1] \longrightarrow H_{0}^{1}(\Omega)$ such that $\varphi(u, t)=u$ if $I(u) \leq 0$, we have that the set $\overline{\varphi(C \times\{1\})}$ is closed and symmetric, for each $C \in \Sigma_{i}$. Moreover, from the supervariancy property of $\gamma^{*}$, it is:

$$
\gamma^{*}(\overline{\varphi(C \times\{1\})})=\gamma^{*}(\varphi(\overline{C \times\{1\}}))=\gamma^{*}(\overline{C \times\{1\}})=\gamma^{*}(C) \geq i,
$$

hence $\overline{\varphi(C \times\{1\})}$ belongs to $\Sigma_{i}$ and the conclusion follows.

So, by Theorem 2.5 there exists $\left.\varepsilon_{2}(j) \in\right] 0, \varepsilon_{1}(j)\left[\right.$ such that, if $|\varepsilon| \leq \varepsilon_{2}(j)$, then $I_{j, \varepsilon}$ has at least $\bar{m}$ odd-essential values $d_{i}^{j, \varepsilon}$, with $i \in\{1, \ldots, \bar{m}\}$, such that

$$
\frac{c_{0}}{2}<d_{1}^{j, \varepsilon}<\ldots<d_{\bar{m}}^{j, \varepsilon}<c_{\infty}+1
$$

As $I_{j, \varepsilon}$ satisfies the Palais-Smale condition in $\mathbb{R}$ (cf. e.g. [1]), by Theorem 2.6, for each $i \in\{1, \ldots, \bar{m}\} I_{j, \varepsilon}$ has a critical point $u_{i}^{j, \varepsilon}$ such that

$$
\left(P_{j, \varepsilon}\right) \quad \begin{cases}-\Delta u_{i}^{j, \varepsilon}-\lambda u_{i}^{j, \varepsilon}=f\left(x, u_{i}^{j, \varepsilon}\right)+\varepsilon g_{j}\left(x, u_{i}^{j, \varepsilon}\right) & \text { in } \Omega, \\ u_{i}^{j, \varepsilon}=0 & \text { on } \partial \Omega\end{cases}
$$

and

$$
d_{i}^{j, \varepsilon}=\frac{1}{2} \int_{\Omega}\left|\nabla u_{i}^{j, \varepsilon}\right|^{2} \mathrm{~d} x-\frac{\lambda}{2} \int_{\Omega}\left(u_{i}^{j, \varepsilon}\right)^{2} \mathrm{~d} x-\int_{\Omega}\left(F\left(x, u_{i}^{j, \varepsilon}\right)+\varepsilon G_{j}\left(x, u_{i}^{j, \varepsilon}\right)\right) \mathrm{d} x .
$$

We claim that

$$
\left\|u_{i}^{j, \varepsilon}\right\| \leq C_{1} \quad \text { for all } j \in \mathbb{N},|\varepsilon| \leq \varepsilon_{2}(j), i \in\{1, \ldots, \bar{m}\} .
$$


Firstly, for all $u \in H_{0}^{1}(\Omega)$, as $u=u^{+}+u^{-}$with $u^{+} \in H^{+}(k+1)$ and $u^{-} \in H^{-}(k)$, standard computations show that there exists $\delta>0$ such that

$$
\begin{aligned}
& \left\|u^{+}\right\|^{2}-\lambda\left|u^{+}\right|_{2}^{2}=\sum_{i=k+1}^{+\infty}\left(\lambda_{i}-\lambda\right)\left|u_{i}\right|_{2}^{2} \geq \delta\left\|u^{+}\right\|^{2}, \\
& \lambda\left|u^{-}\right|^{2}-\left\|u^{-}\right\|_{2}^{2}=\sum_{i=1}^{k}\left(\lambda-\lambda_{i}\right)\left|u_{i}\right|_{2}^{2} \geq \delta\left\|u^{-}\right\|^{2} .
\end{aligned}
$$

Clearly, from $\left(P_{j, \varepsilon}\right)$ and (4.1) we have

$$
\begin{aligned}
& \left\|\left(u_{i}^{j, \varepsilon}\right)^{+}\right\|^{2}-\lambda\left|\left(u_{i}^{j, \varepsilon}\right)^{+}\right|_{2}^{2} \leq \int_{\Omega}\left|f\left(x, u_{i}^{j, \varepsilon}\right)\right|\left|\left(u_{i}^{j, \varepsilon}\right)^{+}\right| \mathrm{d} x+\left|\left(u_{i}^{j, \varepsilon}\right)^{+}\right|_{1}, \\
& \lambda\left|\left(u_{i}^{j, \varepsilon}\right)^{-}\right|_{2}^{2}-\left\|\left(u_{i}^{j, \varepsilon}\right)^{-}\right\|^{2} \leq \int_{\Omega}\left|f\left(x, u_{i}^{j, \varepsilon}\right)\right|\left|\left(u_{i}^{j, \varepsilon}\right)^{-}\right| \mathrm{d} x+\left|\left(u_{i}^{j, \varepsilon}\right)^{-}\right|_{1} .
\end{aligned}
$$

Hence, by (3.1) and (4.4), respectively (4.5), for suitable $\tilde{\varepsilon}, C_{2}>0$, by standard computations we obtain

$$
(\delta-\tilde{\varepsilon})\left\|\left(u_{i}^{j, \varepsilon}\right)^{+}\right\|^{2} \leq \tilde{\varepsilon}\left\|u_{i}^{j, \varepsilon}\right\|^{2}+C_{2}\left\|u_{i}^{j, \varepsilon}\right\|,
$$

respectively

$$
(\delta-\tilde{\varepsilon})\left\|\left(u_{i}^{j, \varepsilon}\right)^{-}\right\|^{2} \leq \tilde{\varepsilon}\left\|u_{i}^{j, \varepsilon}\right\|^{2}+C_{2}\left\|u_{i}^{j, \varepsilon}\right\| .
$$

Putting together the two previous inequalities and choosing $\tilde{\varepsilon}$ small enough, we get that (4.3) holds.

Using regularity results and a standard bootstrap method, we show that from (4.3) we have

$$
\left|u_{i}^{j, \varepsilon}\right|_{\infty} \leq C_{3} \quad \text { for all } j \in \mathbb{N},|\varepsilon| \leq \varepsilon_{2}(j), i \in\{1, \ldots, \bar{m}\} .
$$

Indeed, for $j$ fixed, $u_{i}^{j, \varepsilon} \in L^{2^{*}}(\Omega)$ and so, as $f$ is sublinear, also

$$
\phi_{i}^{j, \varepsilon}\left(x, u_{i}^{j, \varepsilon}\right):=\lambda u_{i}^{j, \varepsilon}+f\left(x, u_{i}^{j, \varepsilon}\right)+\varepsilon g_{j}\left(x, u_{i}^{j, \varepsilon}\right)
$$

belongs to $L^{2^{*}}(\Omega)$. Then, by [17, Theorem B.2], it follows that $u_{i}^{j, \varepsilon} \in H_{0}^{2,2^{*}}(\Omega)$ and

$$
\left\|u_{i}^{j, \varepsilon}\right\|_{H_{0}^{2,2^{*}}} \leq C_{4}\left(\left|u_{i}^{j, \varepsilon}\right|_{2^{*}}+\left|\phi_{i}^{j, \varepsilon}\left(x, u_{i}^{j, \varepsilon}\right)\right|_{2^{*}}\right) .
$$

Then from (4.3) we get

$$
\left\|u_{i}^{j, \varepsilon}\right\|_{H_{0}^{2,2^{*}}} \leq C_{5}
$$

Now, from [17, Theorem A.5], if $N \leq 5,(4.6)$ is true. Otherwise, if $N>6(N=6$ is a simpler case), as $H_{0}^{2,2^{*}}(\Omega)$ is continuously embedded in $L^{q^{*}}(\Omega)$, with $q^{*}=\frac{2 N}{N-6}$, we have that $u_{i}^{j, \varepsilon}$ and $\phi_{i}^{j, \varepsilon}\left(x, u_{i}^{j, \varepsilon}\right)$ are in $H_{0}^{2, q^{*}}(\Omega)$ and, applying again [17, Theorem B.2], we get (4.6) for $N \leq 9$. Going on in this way, (4.6) holds for any $N \in \mathbb{N}$. Finally, for $j>C_{3}$, problem $\left(P_{\varepsilon}\right)$ has at least $\bar{m}$ pairs of solutions.

Remark 4.1. By Remarks 3.2, 3.3 and corresponding suitable changes, the result in Theorem 1.1 still holds if in $\left(H_{2}\right)$ it is $f^{\prime}(0)=0$ or $f^{\prime}(0) \in\{ \pm \infty\}$, with $\bar{m} \geq 1$ number of the distinct critical levels of the unpertubed functional.

Proof of Theorem 1.2. Let us consider the cut functions $\beta_{j}$ and the notations as in the proof of Theorem 1.1. Under our assumptions the functionals $I$ and $I_{j, \varepsilon}$ satisfy $(P S)$ (see [15, Theorem 4.12]). Then $I_{j, \varepsilon}$ has at least $\bar{m}$ odd-essential values $d_{i}^{j, \varepsilon}$ verifying (4.2). Now each $u \in H_{0}^{1}(\Omega)$ can be written as $u=u^{+}+u^{-}+u^{0}$, with 
$u^{+} \in H^{+}(k+1), u^{-} \in H^{-}(k-1)$ and $u_{0} \in M_{k}$. Again standard computations show that there exists $\delta>0$ such that

$$
\begin{aligned}
& \left\|u^{+}\right\|^{2}-\lambda_{k}\left|u^{+}\right|_{2}^{2} \geq \delta\left\|u^{+}\right\|^{2}, \\
& \lambda_{k}\left|u^{-}\right|^{2}-\left\|u^{-}\right\|_{2}^{2} \geq \delta\left\|u^{-}\right\|^{2} .
\end{aligned}
$$

¿From $\left(H_{1}^{\prime}\right),(4.1),\left(P_{j, \varepsilon}\right)$ and $(4.7)-(4.8)$, it follows that

$$
\left\|\left(u_{i}^{j, \varepsilon}\right)^{ \pm}\right\| \leq C_{1} \quad \text { for all } j \in \mathbb{N},|\varepsilon| \leq \varepsilon_{2}(j), i \in\{1, \ldots, \bar{m}\} .
$$

We claim that also $\left\|\left(u_{i}^{j, \varepsilon}\right)^{0}\right\|$ is bounded. Indeed, again $\left(H_{1}^{\prime}\right)$ and standard arguments imply

$$
\left|\int_{\Omega} F(x, u) \mathrm{d} x\right| \leq C_{2}\|u\| \quad \text { for all } \quad u \in H_{0}^{1}(\Omega)
$$

thus, as

$$
I_{j, \varepsilon}\left(u_{i}^{j, \varepsilon}\right) \leq c_{\infty}+1 \quad \text { for all } j \in \mathbb{N},|\varepsilon| \leq \varepsilon_{2}(j), i \in\{1, \ldots, \bar{m}\},
$$

the thesis follows by (4.1) and reasoning as in [15, Theorem 4.12 and Lemma 4.21].

Proof of Theorem 1.3. In this case, once found the critical values of $I$ by Theorem 3.1, as they are assumed to be topologically relevant, we can apply Corollary 2.11 : so there exists $\left.\left.\varepsilon_{2}(j) \in\right] 0, \varepsilon_{1}(j)\right]$ such that, if $|\varepsilon| \leq \varepsilon_{2}(j)$, then $I_{j, \varepsilon}$ has at least $\bar{m}$ critical values $d_{k}^{j, \varepsilon}$, with $k \in\{1, \ldots, \bar{m}\}$, such that (4.2) holds. Then we proceed as in the proof of Theorem 1.1.

Proof of Theorem 1.4. It is enough to combine the arguments in the proofs of Theorems 1.2 and 1.3.

\section{REFERENCES}

[1] H. Amann, E. Zehnder, Nontrivial solutions for a class of nonresonance problems and applications to nonlinear differential equations, Ann. Scuola Norm. Sup. Pisa 7 (1980), 539-603.

[2] A. Ambrosetti, P.H. Rabinowitz, Dual variational methods in critical point theory and applications, J. Funct. Anal. 14 (1973), 349-381.

[3] P. Bartolo, V. Benci, D. Fortunato, Abstract critical point theorems and applications to some nonlinear problems with "strong" resonance at infinity, Nonlinear Anal. 7 (1983), 981-1012.

[4] V. Benci, On the critical point theory for indefinite functionals in the presence of symmetries, Trans. Am. Math. Soc. 274 (1982), 533-572.

[5] V. Benci, A. Capozzi, D. Fortunato, Periodic solutions of Hamiltonian systems with superquadratic potential, Ann. Mat. Pura Appl. CXLIII (1986), 1-46.

[6] J. Cossio, S. Herrón, C. Vélez, Existence of solutions for an asymptotically linear Dirichlet problem via Lazer-Solimini results, Nonlinear Anal. 71 (2009), 66-71.

[7] M. Degiovanni, S. Lancelotti, Perturbations of even nonsmooth functionals, Differential Integral Equations 8 (1995), 981-992.

[8] M. Degiovanni, S. Lancelotti, Perturbations of critical values in nonsmooth critical point theory, in "Well-posed Problems and Stability in Optimization" (Y. Sonntag Ed.), Serdica Math. J. 22 (1996), 427-450.

[9] M. Degiovanni, V. Rădulescu, Perturbations of nonsmooth symmetric nonlinear eigenvalue problems, C.R. Acad. Sci. Paris Sér. I 329 (1999), 281-286.

[10] M.A. Krasnosel'skii, Topological Methods in the Theory of Nonlinear Integral Equations, Translated from the Russian edition, Moscow, 1956 (A.H. Armstrong, J. Burlak Eds), Pergamon, London; Macmillan, New York, 1964.

[11] N. Hirano, W. Zou, A perturbation method for multiple sign-changing solutions, Calc. Var. Partial Differential Equations 37 (2010), 87-98. 
[12] S. Li, Z. Liu, Perturbations from symmetric elliptic boundary value problems, J. Differential Equations 185 (2002), 271-280.

[13] S. Li, Z. Liu, Multiplicity of solutions for some elliptic equations involving critical and supercritical Sobolev exponents, Topol. Methods Nonlinear Anal. 28 (2006), 235-261.

[14] J. Mawhin, M. Willem, Critical Point Theory and Hamiltonian Systems, Springer-Verlag, New York, 1989.

[15] P.H. Rabinowitz, Minimax Methods in Critical Point Theory with Applications to Differential Equations, CBMS Regional Conf. Ser. in Math. 65, Amer. Math. Soc., Providence, 1984.

[16] M. Reeken, Stability of critical points under small perturbations. Part I: Topological theory, Manuscripta Mathematica 7 (1972), 387-411.

[17] M. Struwe, Variational Methods. Applications to Nonlinear Partial Differential Equations and Hamiltonian Systems, 4rd Edition, Ergeb. Math. Grenzgeb. (4) 34, Springer-Verlag, Berlin, 2008.

Rossella Bartolo

Dipartimento di Ingegneria Meccanica e Gestionale

POLITECNICO DI BARI

Via E. Orabona 4, 70125 Bari

ITALY

E-mail address: r.bartolo@poliba.it

Anna Maria Candela and Addolorata Salvatore

Dipartimento di Matematica

Università degli Studi di Bari "Aldo Moro"

Via Orabona 4, 70125 Bari

ITALY

E-mail address: candela@dm.uniba.it

E-mail address: salvator@dm.uniba.it 